\title{
Paralytic poliomyelitis in children under 6 years in Pondicherry: a community survey
}

\author{
M B Soudarssanane, S B Rotti, D K Srinivasa, G Ramalingam
}

\begin{abstract}
Study objectives-To assess the amount of poliomyelitis and its epidemiological features including risk factors.

Design-This was a retrospective study of cases of paralytic poliomyelitis among children 0-6 years of age.

Setting-Pondicherry, India, 1983-89.

Subjects-A total of 47960 children aged less than 6 years.

Measurements and main results-In 1989, 469 field workers undertook a door to door survey of children 0-6 years old to identify those with limb paralysis. This was followed by clinical examination to establish the cause, supplemented by case notes held by the Child Development Services. Altogether 203 cases of limb paralysis were identified, 188 of which were judged a result of paralytic poliomyelitis. The prevalance of poliomyelitis in 1989 was $3 \cdot 9 / 1000$ among children below 6 years of age. There was a male preponderance with a male:female ratio of $1 \cdot 4: 1$. The prevalence was least in infants $(1 / 1000)$ and highest in children aged 2 to 3 years $(6 \cdot 4 / 1000)$. The age at onset was less than 12 months in $42 \%$ of cases and less than 3 years in $98 \%$. The median age at onset was 13.4 months. Time series analysis showed a high occurrence of cases from May to September between 1983 and 1989. The legs were affected in $97 \%$. About $41 \%$ of children had received three doses of oral polio vaccine. There was a history of intramuscular injection, possibly provoking a paralytic attack, in $54 \%$ of cases.

Conclusion-This retrospective community study involving the staff of the Integrated Child Development Services provided valid data about poliomyelitis with little additional cost and minimum training. Because the study covered a whole population of children under 6 years, rather than a sample, the data will help in monitoring and surveillance of poliomyelitis and also in planning strategies for effective control.
\end{abstract}

f Epidemiol Community Health 1993; 47: 210-214

Poliomyelitis continues to be a major public health problem in both urban and rural areas of India. ${ }^{1}$ The number of cases reported is a gross underestimate of the problem. ${ }^{23}$ The information available is based either on cases admitted to hospital or on lameness surveys among children aged $0-5$ and $5-15$ years. There is considerable variation between and within regions. Since the introduction of the Expanded Programme of Immunisation in 1978 and the Universal Immunisation Programme in 1985, efforts have been made to collect information on the incidence of paralytic poliomyelitis. It is essential to try to enlist the support of all available agencies to estimate the extent of the problem and to undertake measures to control it. The Integrated Child Development Scheme (ICDS) which covers about 2500 community development blocks spread over the country offers a good infrastructure for undertaking such activities. The present study was undertaken by all the field staff of the ICDS in Pondicherry, with the objectives of: (a) assessing how much poliomyelitis there is in Pondicherry; and (b) studying the epidemiological features and some risk factors among the cases of paralytic poliomyelitis.

\section{Methods}

This study was conducted in the Pondicherry region of the Union Territory of Pondicherry, situated at a latitude of $11.5 \mathrm{~N}$ along the east coast of the Bay of Bengal and about $160 \mathrm{~km}$ south of Madras. About $74 \%$ of the total population of the Union Territory live here (1981 census). ${ }^{4}$

The vaccination schedule in the study area is as follows: (i) three doses of oral polio vaccine and diphtheria and tetanus in months 3, 4, and 5 of life; (subsequently changed to weeks 6, 10, and 14 under the Universal Immunisation programme; (ii) BCG before 6 weeks of age; and (iii) measles vaccine at 9-12 months of age. Since 1988, vaccination coverage surveys have been conducted every year in Pondicherry in children aged 12-23 months using cluster sampling techniques suggested by Lemeshow and Robinson. ${ }^{5}$ The coverage data for oral polio vaccine were $91 \%$, $97 \%$, and $98 \%$ in 1988, 1989, and 1990 respectively with a very small drop out rate varying from $1 \%$ to $6 \%$ between the first and third doses. A wide network of two major hospitals, 19 primary health centres, and nine dispensaries carry out the vaccination programmes. The peripheral institutions undertake immunisation on a fixed day of the week throughout the region using outreach operations.

The Integrated Child Development Scheme (ICDS) has four projects in Pondicherry with a total of 469 anganwadis (field workers) as of June 1989. The activities of the ICDS have already been documented. ${ }^{6}$ There is one anganwadi worker for about one thousand population. During the monthly continuing education sessions in April, May, and June 1989, all anganwadi workers, project supervisors, and child development project officers were briefed in detail about the objectives of the study and the methodology. 
All the anganwadi workers conducted a door to door survey of the children with limb paralysis in the age group 0-6 years in their respective area. Data available from the ICDS records were also used to support the information gathered in the survey. A total of 47960 children formed the study group. This study was undertaken in AprilJuly 1989 . Data were collected by the anganwadi worker using a proforma that contained information about identification data, age, sex, age at the onset of paralysis, and the risk factors of poliomyelitis cases along with the age and sex distribution of all children under 6 years in their respective area. The following criteria, which were used for the lameness survey under the Universal Immunisation Programme, were used for this study:

(i) History of fever for one to two days preceding the onset of weakness or paralysis of the muscles of the limbs;

(ii) No progress of the paralysis after the first three days;

(iii) Paralysis was not present at birth;

(iv) No sensory loss.

A total of 203 cases were identified by the workers. All were referred to the Centre for Education, Rehabilitation, and Treatment of the Handicapped (CERTH) in Pondicherry using transport provided by the Department of Social Welfare. The diagnosis of paralytic poliomyelitis was confirmed at CERTH by a team of doctors, which included one of the authors (MBS). Altogether 188 cases of lameness were confirmed to have been caused by paralytic poliomyelitis: 11 were the result of neurological sequelae of encephalitis, two were caused by congenital deformity, and two by tuberculous meningitis. Whenever these were available, discharge slips given by the treating hospitals were referred for the diagnosis.

Laforce $e t a l^{7}$ have suggested that the figures for lameness should be multiplied by a factor of 1.25 to arrive at the total number of paralytic cases. The

Table I Prevalence of paralytic poliomyelitis 11000 children under 6 years of age in Pondicherry

\begin{tabular}{|c|c|c|c|}
\hline \multirow[b]{2}{*}{ Age $(y)$} & \multicolumn{3}{|c|}{ Prevalence /1000 children $<6 y$} \\
\hline & Boys & Girls & Total \\
\hline 1 & $1 \cdot 3$ & 0.8 & 1.0 \\
\hline 2 & $2 \cdot 7$ & 1.9 & $2 \cdot 3$ \\
\hline 3 & $7 \cdot 9$ & $4 \cdot 9$ & $6 \cdot 4$ \\
\hline 4 & $4 \cdot 6$ & $3 \cdot 8$ & $4 \cdot 2$ \\
\hline 5 & $5 \cdot 5$ & $4 \cdot 4$ & 4.9 \\
\hline 6 & $5 \cdot 3$ & $3 \cdot 8$ & $4 \cdot 5$ \\
\hline Total & $4 \cdot 6$ & $3 \cdot 3$ & 3.9 \\
\hline
\end{tabular}

Figure 1 The occurrence of poliomyelitis in Pondicherry between 1983 and 1989. product should then be multiplied by a factor of 1.33 to account for cases that might have either recovered or died. To arrive at the incidence this total should be divided by 5 if the age group at risk in the study population is $0-5$ years. In this study, multiplication of the number of cases identified by 1.25 was not done as children with affected upper limbs were also included. The total prevalence was divided by 6 as the age group at risk was $0-6$ years, to arrive at the yearly incidence.

Time series analysis for the period July 1983 to June 1989 was done using the method of moving averages. The average of three consecutive months was calculated omitting the first month each time and taking the average value as the expected value for the middle month. For example the average of three months of 1983, July, August and September, was taken to be the expected value for August 1983. The average for the next set of three months August, September, and October 1983 was calculated to give the expected value for September 1983. The expected values for each month in the study period were calculated similarly. The average of all the expected values for the same month for all the years from 1983 to 1989 was calculated next - that is, the average of all the expected January values for 1983 to 1989 , and so on. Thus, we calculated the individual average for 12 months, which formed the average year. The mean value of 12 months of the average year formed the ' $\mathrm{O}$ ' in the $\mathrm{X}$ axis. Values on the positive and negative side of ' $O$ ' were plotted on the $\mathrm{Y}$ axis. This procedure of calculating time trends is fully described by S P Gupta. ${ }^{8}$

\section{Results}

The prevalance of poliomyelitis in children under 6 years of age in 1989 was 3.9/1000 (188 cases among 47 960) in Pondicherry. It was least among infants. The maximum prevalence was among those aged 2 years. It was two to three times higher in children aged 2 years and above than in those below 2 years of age (table I). The difference was statistically significant $\left(\chi^{2}=30 \cdot 18 ; p<0 \cdot 001\right)$.

There were 109 cases in boys, giving a prevalence of $4 \cdot 6 / 1000$, and 79 cases in girls, giving a prevalence of 3.3/1000. The boy:girl ratio was $1 \cdot 4: 1$, and the difference was statistically significant $\left(\chi^{2}=5 \cdot 21 ; p<0 \cdot 05\right)$.

The prevalence of all cases of poliomyelitis was $5 \cdot 2 / 1000$ children after the correction factor of 1.33 was applied. The annual incidence was calculated to be $0 \cdot 9 / 1000$. The yearwise occurrence of poliomyelitis (calculation based on the year of onset) showed a maximum occurrence in 1987. Of the total of 188 cases, there were 4 in 1989 (up to June), 24 in 1988, 64 in 1987, 36 in 1986, 30 in 1985, 25 in 1984, and 5 in 1983 (from July) (fig 1).

The incidence of poliomyelitis was calculated using figures for the new occurrences every year and the total number of children under 6 years served by the ICDS for the corresponding years. The incidence rates (per 1000 for children under 6 years were 0.85 in $1984,0.74$ in $1985,0.82$ in $1986,1.51$ in 1987 , and 0.57 in 1988 . (fig 2)

The age at onset of paralytic poliomyelitis is shown in table II. Infants aged less than 6 months contributed $13.3 \%$ and about $75 \%$ of the cases 
Figure 2 The incidence of poliomyelitis in Pondicherry between 1984 and 1988.

Table II Frequency of paralytic poliomyelitis by age at onset

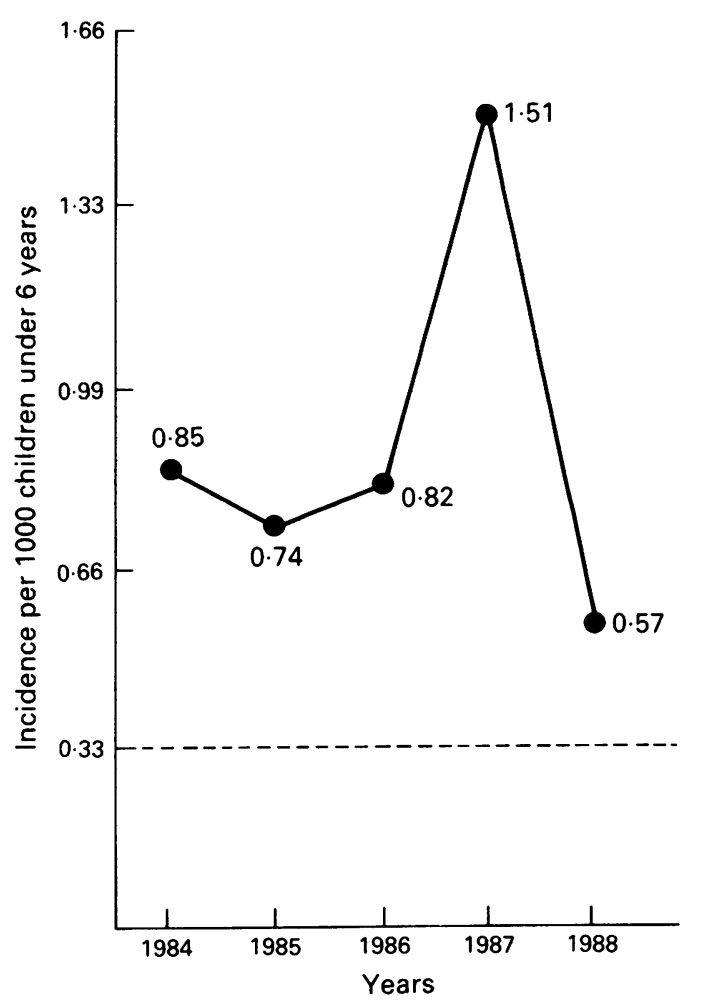

\begin{tabular}{lll}
\hline Age at onset (mth) & $\begin{array}{l}\text { Frequency (\%) } \\
(n=188)\end{array}$ & $\begin{array}{l}\text { Cumulative frequency } \\
(\%)\end{array}$ \\
\hline$<6$ & $13 \cdot 3$ & $13 \cdot 3$ \\
$6-12$ & $28 \cdot 2$ & $41 \cdot 5$ \\
$13-18$ & $35 \cdot 6$ & $77 \cdot 1$ \\
$19-24$ & $8 \cdot 5$ & $85 \cdot 6$ \\
$25-36$ & $12 \cdot 2$ & $97 \cdot 8$ \\
$37-60$ & $2 \cdot 2$ & $100 \cdot 0$ \\
\hline
\end{tabular}

had occurred before the age of 18 months. In all cases, the age at onset was less than 5 years. The median age at onset was 13.4 months (fig 3).

Limb involvement is shown in table III. Legs were affected in $97.3 \%$ and arms in $2.7 \%$ of cases.

Risk factors for paralytic poliomyelitis are given in table IV. About $60 \%$ of cases were either not immunised or only partially immunised and there was a history of injection during the period of fever before paralysis in $54 \%$ of cases.

Time series analysis using the month of onset showed a seasonal distribution of cases, from May to September (fig 4), though there were scattered occurrences throughout the year.

\section{Discussion}

Developed countries have reached the stage of eliminating the occurrence of new cases and are embarking upon eradication of poliomyelitis ${ }^{9}$ but the disease is still endemic in developing countries, which experience seasonal and cyclical outbreaks. ${ }^{10}$ In India the Universal Immunisation Programme was launched in 1985 to increase immunisation coverage for the six vaccine preventable diseases of childhood and to train the health personnel. The field staff of the Integrated Child Development Services are being increasingly involved in the activities of the Universal Immunisation Programme. In the present study the infrastructure of ICDS was used and this offered the advantage of accurately specifying the characteristics of the population under study. The study group was the total population aged under 6 years rather than a sample. The procedure entailed little extra time and cost to the investigators. Moreover, cases studied included all forms of paralysis caused by poliomyelitis, including lameness.

The methodology employed in the present study was the same as that used for conducting lameness surveys for poliomyelitis under the Universal Immunisation Programme. ${ }^{5}$ In such surveys carried out in Pondicherry, the information was collected retrospectively, with the recall period varying from zero to five years. ${ }^{11}{ }^{12}$ Some previous studies have also reported retrospective data. ${ }^{13-15}$ Some recall bias cannot be ruled out in these studies (including the present study) but they have given data comparable with those of some hopsital studies $^{1617}$ in which the recall bias could be expected to be less. Prospective studies would be ideal but they would be costlier and more time consuming.

The estimated annual incidence of poliomyelitis in this study was $0 \cdot 9 / 1000$, which agrees with the overall incidence of $1-2 / 1000$ in India. ${ }^{2}$ The target is to reduce the incidence of poliomyelitis to $0 \cdot 33 / 1000$ children age less than 5 years by 1990 . In this study it was found that the incidence was $0 \cdot 57 / 1000$ children under 6 years of age in 1988 .

The prevalence of lameness as a result of paralytic poliomyelitis was $1 \cdot 6 / 1000$ children aged $6-15$ years in the early 1980 s in Pondicherry. ${ }^{15}$

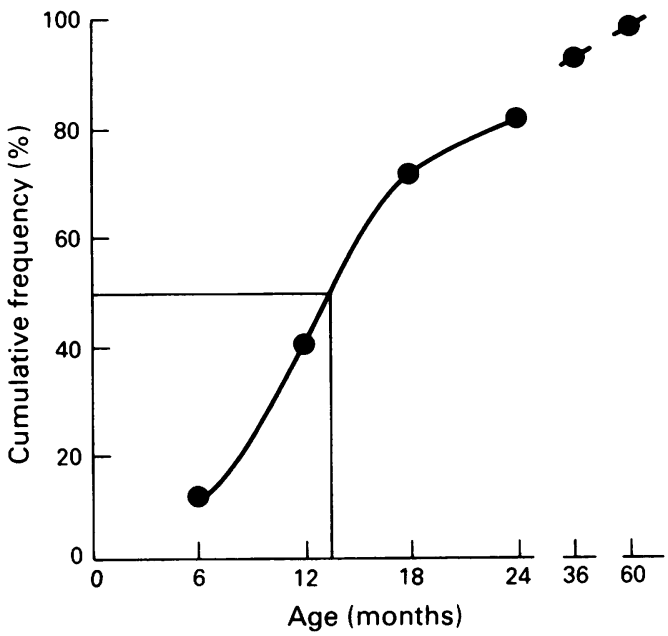

Figure 3 Cumulative frequency of the age distribution and median age at onset of poliomyelitis.

Table III Limb involvement among paralytic

poliomyelitis cases

\begin{tabular}{ll}
\hline Limb involved & $\begin{array}{l}\text { \% of cases } \\
(n=188)\end{array}$ \\
\hline Lower limbs: & $97 \cdot 3$ \\
Left alone & $40 \cdot 4$ \\
Right alone & $29 \cdot 3$ \\
Both & $27 \cdot 6$ \\
Upper limbs: & $2 \cdot 7$ \\
Left alone & $1 \cdot 6$ \\
Right alone & $1 \cdot 1$ \\
\hline
\end{tabular}

Table IV Frequency of risk factors for paralytic poliomyelitis $(n=188)$

\begin{tabular}{lrl}
\hline Immunisation status: & No & (\%) \\
\hline Unimmunised or partly immunised & 110 & $(59 \cdot 5)$ \\
Fully immunised & 78 & $(40 \cdot 5)$ \\
History of injection: & 102 & $(54 \cdot 2)$ \\
Yes & 86 & $(45 \cdot 8)$ \\
No & & \\
\hline
\end{tabular}




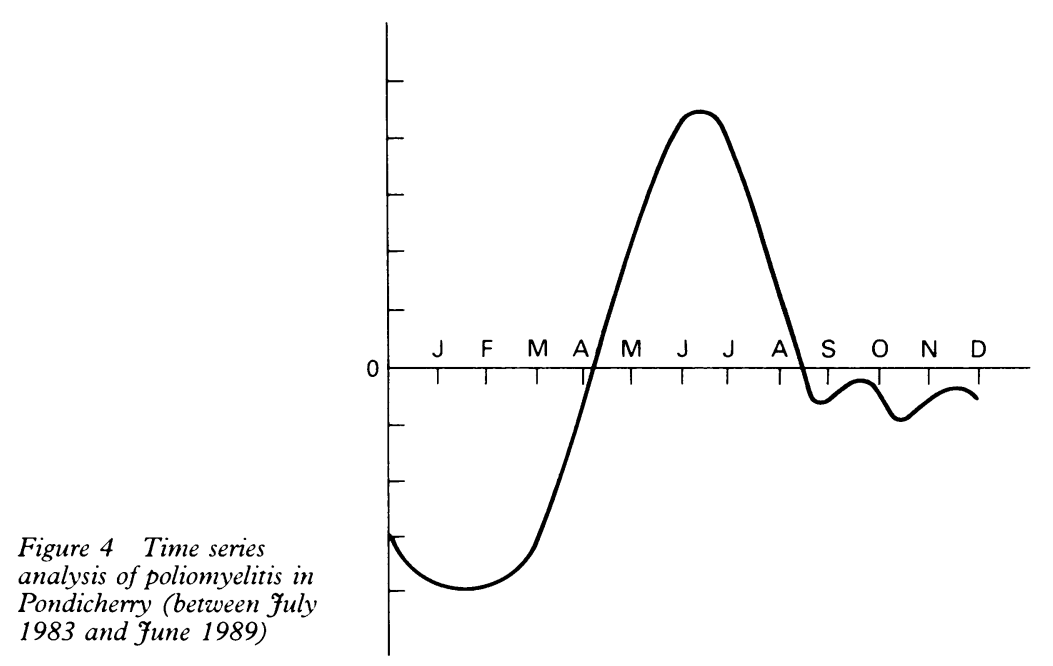

The prevalence of paralytic poliomyelitis of 3.9/ 1000 children under 6 years of age in this study indicates that the occurrence of new cases is concentrated in preschool children and reflects a rise in the occurrence in the past decade.

There is evidence for the occurrence of outbreaks of poliomyelitis in 1987 in Pondicherry and adjoining districts of Tamil Nahu. ${ }^{16}$ This is reflected in our study too as the maximum prevalence was among children aged $2-3$ years, corresponding to the year 1987. Again, the incidence in 1987 was as high as $1 \cdot 51 / 1000$, compared with a value below $0 \cdot 8 / 1000$ in the other years in the study, denoting the epidemic in 1987. Cluster sample surveys conducted in Pondicherry in 1989 and 1990 have reported prevalences of lameness caused by poliomyelitis as $4 \cdot 6$ and $2 \cdot 4 / 1000$ respectively in children under 5 years of age. ${ }^{11} 12$ Other studies conducted in rural and urban areas of south India have also indicated a rise in the last decade. ${ }^{141822}$

The preponderance of cases among males observed in this study has also been reported in the studies conducted in Pondicherry and elsewhere. ${ }^{15} 1623-24$

In a series of cases admitted in hospital over the past 20 years in Pondicherry ${ }^{16}$ and in two field surveys recently conducted in Pondicherry, ${ }^{11} 12$ the age at onset was reported to be under 12 months in most $(50 \%-62 \%)$ cases. In the present study too age of onset was less than 12 months in $41.5 \%$ of cases. The median age at onset has been reported to be 11-12 months. ${ }^{16}{ }^{19}$ The present study also confirms this finding. In addition, the fact that $13 \%$ of cases have occurred in children below the age of 6 months underlines the importance of the zero dose of oral polio vaccine and completion of the primary vaccination of three doses before 6 months of age.

The legs were affected in $97 \%$ of cases in the present study. Other studies have reported paralysis affecting the legs in $75-90 \%$ of cases. ${ }^{23} 26$

The frequency of children developing paralytic poliomyelitis even after receiving three doses of oral polio vaccine has been high in Pondicherry: it was $58 \%$ in a cluster sample survey in $1990^{12}$ and $14.7 \%$ in a hospital study. ${ }^{16}$ In the present study it was $40 \cdot 5 \%$. Since the vaccine coverage for all the children under 6 years of age included in the study was not known, it is not possible to comment on the vaccine efficacy. Failure of oral polio vaccine has been reported to range between 5 and $20 \% .^{27}$
The causes of the apparent higher failure of oral polio vaccine in our study need further investigations to find if other factors are involved.

The results of this study show that intramuscular injection may have provoked poliomyelitis in $54 \%$ of the cases. Mahadevan et $a l^{16}$ have also reported provocation poliomyelitis in $66 \%$ of cases. Several reports from India $202+27$ and other third world countries ${ }^{28-30}$ have indicated that intramuscular injections given for non-specific fever in children are an important risk factor in areas that are endemic for poliomyelitis.

In the Indian subcontinent, the peak incidence has been reported during July, August, and September. ${ }^{1}$ The present study also confirms this observation with a slightly extended duration from May to September. This calls for intensification of the immunisation programme during the low polio incidence period of November to April.

We are indebted to The Director of Social Welfare and the Programme Officer, ICDS Cell, Government of Pondicherry for their kind cooperation. We thank all the CDPOs, programme supervisors, and anganwadi workers of ICDS projects of Pondicherry region for their active participation in this study. We also thank the Director of JIPMER, Pondicherry, Dr D S Dubey for his encouragement, the Director, Centre for Education Rehabilitation, and Treatment of the Handicapped (CERTH), Pondicherry, Mr Marc Bonnet, for providing calipers and other appliances to all the identified children; Dr S Mahadevan for his invaluable help in the literature search; Mrs C Maragathamani and Mr G K Padmanabhan for their secretarial assistance.

1 World Health Organisation. The EPI in SE Asia, WHO: New Delhi, 1986. SEARO Reg Health Paper No 12.

2 Basu RN. Magnitude of problem of poliomyelitis in India. Indian Pediatr 1981; 18: 507-11.

3 Health information of India. New Delhi: Government of India Central Bureau of Health Intelligence, 1986

4 Abstracts of statistics (1982-83), Pondicherry: Director of Economics and Statistics, 1984

5 Lemeshow S, Robinson D. Survevs to measure programme coverage andimpact: a review of the methodology used by the expanded programme on immunisation, World Health Stat $Q 1985 ; 38: 65-75$.

6 Central Technical Committee on health and nutrition. Integrated Child Development Services New Delhi: India Institute of Medical Sciences, 1983. (Information document)

7 LaForce FM, Lichnevski MS, Keja J, Henderson RH. Clinical survev technique to estimate prevalance and annual incidence of poliomyelitis in developing countries. Bull incidence of poliomyelitis in developing
World Health Organ 1980; 58 (4);609-20.

8 Gupta SP. Statistical methods (25th ed). New Delhi: Sultan Chand, 1990, 18-24.

9 World Health Organisation Weekly Epidemiological Records. 1981: 56 (43); 337-44.

10 World Health Organisation Weekly Epidemiological Records 1976: 51; 53-60.

11 Report on the immunisation coverage, antenatal care, KAP and lameness survey in Pondicherry. Pondicherry, Jawaharlal Institute of Postgraduate Medical Education and Research 1989.

12 Report on the immunisation coverage, antenatal care, KAP, neonatal deaths, and lameness survey in Pondichery. Pondicherry, Jawaharlal Institute of Postgraduate Medical Education and Research: 1990.

13 Jhala CI, Goela RK, Dave SK, Dave AD. Morbidity due to poliomyelitis in urban and rural area of Gujarat in pediatric population-a house-to-house survey. Indian Pediatr 1976; 13: $821-5$.

14 Prabhakar N, Srilatha V, Mukerii D ct al. The epidemiology and prevention of poliomyelitis in a rural community in south India. Indian Pediatr 1981; 18: 527-32.

15 Rotti SB, Satpathy SK, Mehta SP. Prevalence of paralytic poliomyelitis in Pondicherry, South India. $\mathcal{f}$ Epidemiol Community Health 1982: 36; 279-81.

16 Mahadevan S, Shanti A, Srinivasan S. Poliomyelitis. 20 years-the Pondicherry experience. F Trop Med Hyg 1989; 92: 416-21.

17 Gujral V, Sharma D, Gangrade S, et al. Paralytic poliomyelitis in children. Indian Pediatr 1977: 14; 379-85. 18 Sundaravalli N, Narmada R, Sankar, et al. Spurt of poliomyelitis in Madras. Indian Pediatr 1981: 18; $539-44$. 19 Maiya PP, Jadhav $M$, Mukundan $P$, et al. Paralytic poliomyelitis-clinical and virological observations. Further study on 210 children. Indian Pediatr 1981; 18: 533-7.

$20 \mathrm{John} \mathrm{TJ}$, John TK. Is poliomyelitis a serious problem in developing countries? The Vellore experience. $f$ T Top Pediat 1982: 28; 11-13. 
21 Vijayan P, Mukundan P, Sukhoor AA, et al. Epidemic poliomyelitis. Indian Pediatr 1985: 22; 569-73.

22 Santhanakrishan BR, Deivanayagam N, Parthasarathy A, et al. Poliomyelitis in children. Indian foumal of Pediatrics 1987: 54: 755-58.

23 Pendey S, Alam B, Jhe SS, et al Polioparalysis. A critical review, fournal of Rehabilitation in Asia 1975: 20: 21-7.

24 Sancheti KH, Sahasrabudhe BG, Bhingare RK, Electricwala JT. Clinico-environmental profile of residual paralytic poliomyelitis. Indian Council of Medical Research Bulletin 1981; 2: 59-66.

25 Sahadeva KC, Gupta AC. Polio paralysis. Clinical observations and management report of 500 cases. fournal of Rehabition in Asia 1972, 13: 7-11.
26 Punatar BJ, Patel DA. Pattern of residual paralysis in poliomyelitis. Indian fournal of Orthopaedics 1971: 2; 174-8.

27 Johns TJ. Poliomyelitis in India. Prospects and problems in control. Rev Infect Dis 1984; 6 (suppl 2): 438-41.

28 Nicholas DD, Kratzer JM, Ofasu Ammah S, et al. Is poliomyelitis a serious problem in developing countres? The Dafna experience. BMf 1977, i: 1009-12.

29 Nwuga VCB, Odunowa T. Some clinical characteristics of children with paralytic poliomyelitis referred for physiotherapy. 7 Trop Med Hyg 1978, 81: 84-7.

30 Guyer B, Bisong AAE, Gould J, et al. Infections and paralytic Poliomyelitis in tropical Africa. Bull World Health Organ 1980: 58; 285-91.

\section{An 18th century trial}

Sir Austin Bradford Hill stated ${ }^{1}$ that the essence of any trial is comparison and gave the following quotation from the writings of James Lind $(1753)^{2}$ as an example of a classical experiment in the treatment of scurvy which makes clear this concept.

"On the 20th of May, 1747, I took twelve patients in the scurvy, on board the Salisbury at sea. Their cases were as similar as I could have them. They all in general had putrid gums, the spots and lassitude, with weakness of their knees. They lay together in one place, being a proper apartment for the sick in the fore-hold; and had one diet common to all, viz. water-gruel sweetened with sugar in the morning; fresh mutton-broth often times for dinner; at other times puddings, boiled biscuit with sugar etc. And for supper, barley and raisins, rice and currants, sago and wine, or the like. Two of these were ordered each a quart of cyder a day. Two others took twenty-five gutts of elixir vitriol three times a day, upon an empty stomach; using a gargle strongly acidulated with it for their mouths. Two others took two spoonfuls of vinegar three times a day, upon an empty stomach; having their gruels and their other food well acidulated with it, as also the gargle for their mouths. Two of the worst patients, with the tendons in the ham rigid (a symptom none of the rest had) were put under a course of sea-water. Of this they drank half a pint every day, and sometimes more or less as it operated, by way of gentle physic. Two others had each two oranges and one lemon given them every day. These they eat with greediness, at different times, upon an empty stomach. They continued but six days under this course, having consumed the quantity that could be spared. The two remaining patients, took the bigness of a nutmeg three times a day of an electuary recommended by a hospital-surgeon, made of garlic, mustard-seed, rad. raphan, balsam of Peru, and gum myrrh; using for common drink barley-water well acidulated with tamarinds; by a decoction of which, with the addition of cremor tartar, they were greatly purged three or four times during the course.

The consequence was, that the most sudden and visible good effects were perceived from the use of the oranges and lemons; one of those who had taken them, being at the end of six days fit for duty. The spots were not indeed at that time quite off his body, nor his gums sound; but without any other medicine, than a gargle of elixir vitriol, he became quite healthy before we came into Plymouth, which was on the 16 th June. The other was the best recovered of any in his condition; and being now deemed pretty well, was appointed nurse to the rest of the sick."

1 Hill AB. Statistical methods in clinical and preventive medicine. London: Livingstone, 1962.

2 Lind J. A treatise of the scurvy in three parts, containing an inquiry into the nature, causes and cure of that disease, together with a critical and chronological view of what has been published on the subject. Edinburgh: Sands, Murray, and Cochran, 1753. 